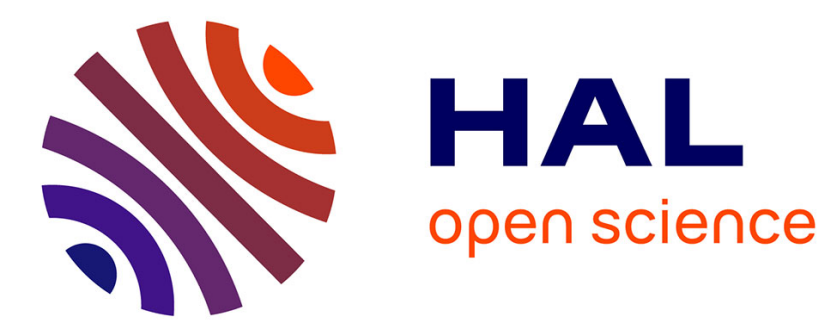

\title{
A finite volume method to solve the 3D Navier-Stokes equations on unstructured collocated meshes
}

\author{
Sébastien Perron, Sylvain Boivin, Jean-Marc Hérard
}

\section{To cite this version:}

Sébastien Perron, Sylvain Boivin, Jean-Marc Hérard. A finite volume method to solve the 3D NavierStokes equations on unstructured collocated meshes. Computers and Fluids, 2004, 33 (10), pp.13051333. 10.1016/j.compfluid.2003.10.006 . hal-01291273

\section{HAL Id: hal-01291273 \\ https://hal.science/hal-01291273}

Submitted on 24 Mar 2016

HAL is a multi-disciplinary open access archive for the deposit and dissemination of scientific research documents, whether they are published or not. The documents may come from teaching and research institutions in France or abroad, or from public or private research centers.
L'archive ouverte pluridisciplinaire HAL, est destinée au dépôt et à la diffusion de documents scientifiques de niveau recherche, publiés ou non, émanant des établissements d'enseignement et de recherche français ou étrangers, des laboratoires publics ou privés. 


\title{
A new finite volume method to solve the 3D Navier-Stokes equations on unstructured meshes
}

\author{
Sébastien Perron ${ }^{1}$, Sylvain Boivin ${ }^{1}$, Jean-Marc Hérard ${ }^{2}$, \\ ${ }^{1}$ Université du Québec à Chicoutimi, 555 Boulevard de l'université, Chicoutimi, Québec Canada g7H 2 B1. \\ ${ }^{2}$ Direction des études de recherches, Électricité de France, Chaton, France.
}

31st March 2002

\begin{abstract}
A new method to solve the Navier-Stokes equations for incompressible viscous flows and the transport of a scalar quantity is proposed. This method is based upon a fractional time step scheme and the finite volume method on unstructured meshes. Predictions of Poiseuille flow, lid-driven cavity flow, flows past a cylinder and heat transport in a cylinder are performed to validate the method.
\end{abstract}

\section{Introduction, Mathematical model}

Many industrial problems deal with the transport of a scalar quantity by an incompressible flow. These problems are usually governed by Navier-Stokes equations and are often coupled with additional equations to model the transport of scalar quantities such as a pollutant, a volume fraction or the kinetic energy. Even though many stable and globally convergent schemes are already available for specific cases such as Euler flows or Stokes flows, only a few fulfill the following physical principles for more general problems:

- local conservation of mass and scalar quantities;

- numerical preservation of the maximum principle for the scalar quantities.

Some numerical schemes using the finite volume method on structured meshes satisfy these requirements. However, only a few results are available for unstructured meshes (see Gallouet and al.[8]). The scheme presented hereafter fulfills the previous requirements. The time discretization of this scheme is based upon a fractional time-step method initially proposed by Chorin[7] and 
theoretical results more recently published by Boivin and Hérard[3]. The spatial discretization is a generalization of the recent work of Boivin and al. (see [1], [2] and [6]).

When the density of the fluid is constant, the flow of an incompressible fluid in a domain $\Omega \times[0, T]$ is governed by the Navier-Stokes equations:

$$
\begin{aligned}
\nabla \cdot \underline{v} & =0 \\
\frac{\partial \underline{v}}{\partial t}+\nabla \cdot(\underline{v} \otimes \underline{v})+\nabla P-\nabla(v \nabla \underline{v}) & =\underline{f}
\end{aligned}
$$

where

- $P=p / \rho$, with $\rho$ being the density and $p$ the pressure;

- $v=\mu / \rho, v$ being the kinematic viscosity and $\mu$ the dynamic viscosity;

- $\underline{f}$ is a source term such as buyoancy force.

For this kind of flow, a model for the transport of a scalar quantity $\phi$ is:

$$
\frac{\partial \phi}{\partial t}+\nabla \cdot(\underline{v} \phi)-\nabla(\alpha \nabla \phi)=s
$$

where $\alpha$ is a physical parameter for the diffusivity and $s$ a source term.

In order to solve these equations s they must come with appropriate boundary conditions and for non-permanent flow, a suitable initial solution. This model can be extended to many applications such as heat transfer or the transport of a pollutant by adjusting the source terms to the problem being considered.

First of all, we will present the time discretization of the mathematical model. Then, the spatial discretization of the governing equations will be detailed and some theoretical results will be presented. Afterwards, we will discuss the projection scheme used to compute a divergence-free vector field. This will enable the presentation of the whole algorithm used to solve the Navier-Stokes equations on unstructured grids. Finally, numerical results used to validate the present scheme will be shown. These results include: Poiseuille flow, lid-driven cavity flow, both permanent and transient flow past a cylinder and heat transport in a cylinder.

\section{Time discretization}

The time discretization is semi-implicit and based upon a variation of the projection scheme originally proposed by Chorin[7] and often called "projection-2" scheme. Let,

$$
\left.\frac{\partial \phi}{\partial t}\right|_{t=t_{n+1}}=\left\{\begin{array}{cc}
\frac{\phi^{n+1}-\phi^{n}}{\delta t}, & \text { permanent flow } \\
\frac{3 \phi^{n+1}-4 \phi^{n}-\phi^{n-1}}{2 \delta t}, & \text { otherwise }
\end{array}\right.
$$


be the approximation of the temporal derivative and

$$
\underline{v}\left(t=t_{n+1}\right)=\left\{\begin{array}{cc}
\frac{3}{2} \underline{v}^{n}-\frac{1}{2} \underline{v}^{n-1} & \text { momentum equations, permanent flow } \\
2 \underline{v}^{n}-\underline{v}^{n-1} & \text { momentum equations, transient flow } \\
\underline{v}^{n+1} & \text { otherwise }
\end{array}\right.
$$

the approximation of the velocity field at time $t=t_{n+1}$, the time discretization of the governing equations is the following:

- Prediction:

$$
\left.\frac{\partial \underline{v}}{\partial t}\right|_{t=t_{n+1}}+\nabla \cdot\left[\underline{v}\left(t=t_{n+1}\right) \otimes \underline{v}^{n+1 / 2}-v \nabla \underline{v}^{n+1 / 2}\right]+\nabla P^{n}=\underline{f}^{n} .
$$

- Projection:

$$
\begin{aligned}
\beta \frac{\underline{v}^{n+1}-\underline{v}^{n+1 / 2}}{\delta t} & =-\nabla\left(\delta P^{n+1}\right), \\
\nabla \cdot \underline{v}^{n+1} & =0 .
\end{aligned}
$$

- Convection and diffusion of other scalar variables:

$$
\left.\frac{\partial \phi}{\partial t}\right|_{t_{n+1}}+\nabla \cdot\left[\underline{v}\left(t_{n+1}\right) \phi^{n+1}-\alpha \nabla \phi^{n+1}\right]=s^{n} .
$$

The parameter $\beta \in] 0,2]$ is a used for relaxation although its value is not arbitrary. When the flow is permanent, the final solution doesn't depend on its value. But the rate of convergence does so. We found that a value of $\beta=\frac{3}{2}$ often gives the best rate of convergence. Hence this value is the one retained for all stationary results shown in this article. For a transient flow, the value $\frac{1}{2}$ was chosen accordingly with the results published by Shen and al. on projection schemes (see [10], [15] and [14]). These authors showed that second order time accuracy for the velocity can be reached with this class of projection scheme. Unfortunalty, the time accuracy for the pressure is only first order. For a transient flow, the linear parabolic operator is approximated with a second-order backward scheme often called "Gear's scheme". As for the non-linear convective terms, a semi-implicit time discretization is prefered: the convective field in the momentum equations is extrapolated with the Adams-bashworth scheme when a stationary solution is seeken. In the case of a transient flow, the Adams-Moulton scheme is prefered. 


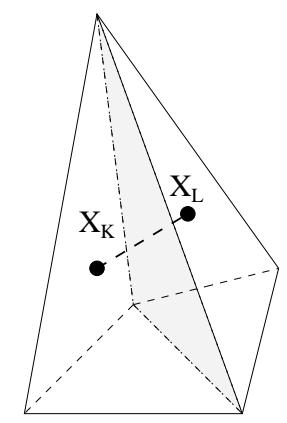

Figure 1: Circumcenters for tetrahedras $K$ and $L$.

\section{Space discretization}

The convection and diffusion schemes are presented thereafter. The convection scheme is simple and robust. The diffusion scheme is based upon theoretical results recently published (see [6], [1] and [8]). A numerical study of this diffusion scheme was performed in the report [5]. Finally, in $2 \mathrm{D}$, this numerical scheme has given good results for the resolution of incompressible two-phase flows (see [6] and [2]).

\subsection{Geometrical elements}

The initial mesh is built with tetrahedras. For each tetrahedron $T_{K}$, let $X_{K}$ be the circumcenter. This point is allowed to be inside $T_{K}$, outside $T_{K}$, or on its boundary. Let $\sigma_{K, L}$ be the interface between $T_{K}$ and $T_{L}$, the straight line going through $X_{K}$ and $X_{L}$ is always perpendicular to $\sigma_{K, L}$ (figure 1). Hence, in order to have a consistent approximation of the diffusion flux with a small stencil, the circumcenters will be the reference positions for the numerical approximation of the fluxes.

\subsection{Control volumes}

Let $\sigma_{K, L}$ be the interface between tetrahedras $K$ and $L$, we then introduce the following quantity called "transmittivity":

$$
\tau_{K, L}=\frac{m\left(\sigma_{K, L}\right)}{\left(X_{L}-X_{K}\right) \cdot \underline{n}_{K, L}},
$$

where $m\left(\sigma_{K, L}\right)$ is the area of $\sigma_{K, L}$. Locally, four categories of meshes can be encountered:

1. Categories $M_{1}$ et $M_{2}: \tau_{K, L}>0$, the control volumes are the tetrahedras.

2. Category $M_{3}: \tau_{K, L}<0$, the Delaunay condition is not fulfilled. The tetrahedron $K$ and $L$ are combined to form a new macro-element. The control volume is the macro-element. At least two positions $\left(X_{K}\right.$ and $\left.X_{L}\right)$ are associated to this control volume. 


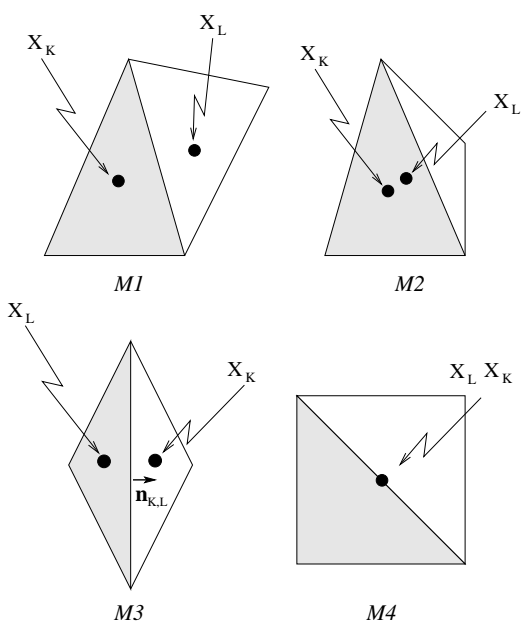

Figure 2: Mesh categories

3. Category $M_{4}:\left|\tau_{K, L}\right| \rightarrow \infty$, at least two circumcenters lie at the same location. The elements $K$ and $L$ are combined to form a new macro-element. The control volume is the macro-element. At least two positions $\left(X_{K}\right.$ and $\left.X_{L}\right)$ are associated to this control volume.

These categories are the same in 2D when triangles are the elements, they are shown in figure 2.

\subsection{Approximation functions}

For all variables, piecewise constant functions by control volumes are used for approximation. Even when there are more than one position associated to a control volume (meshes of categories $M_{3}$ and $M_{4}$ ), there is only one degree of freedom (D.O.F.) or unknown per control volume.

\subsection{The convection scheme}

First, the convective term is integrated. Then, the divergence theorem is applied to the convection term. The convection flux is approximated with an upwind scheme. The discrete approximation of the total convection flux between volumes $K$ and $L$ is:

$$
\int_{K, L}(\underline{v} \phi(\mathbf{x})) \cdot \underline{n}(\underline{x}) d S \approx m\left(\sigma_{K, L}\right) v_{\sigma} \phi_{\sigma,+}=F_{\sigma}^{C}
$$

where:

- $v_{\sigma}$ is an approximation of the speed normal to the interface $\sigma, \underline{n}(\underline{x})$ is the outward normal unit vector;

- $\phi_{\sigma,+}=\left\{\begin{array}{ll}\phi_{K}, & v_{K, L} \geq 0 \\ \phi_{L}, & \text { otherwise }\end{array}\right.$, 
$\phi_{K}$ and $\phi_{L}$ are the unknowns associated with volumes $K$ and $L$.

\subsection{The diffusion scheme}

The diffusion term is integrated and the divergence theorem applied. Then, the total diffusion flux between volumes $K$ and $L$ is approximated with the following expression:

$$
\int_{K / L} \alpha \nabla \phi(\underline{x}) \cdot \underline{n}(\underline{x}) d S \approx \alpha_{K, L} \tau_{K, L}\left(\phi_{L}-\phi_{K}\right)=F_{\sigma}^{D},
$$

where $\alpha_{K, L}$ is a discrete approximation of the diffusivity $\alpha(\mathbf{x})$ a the interface $\sigma_{K . L}$. We recall that $\tau_{K, L}$ is the transmittivity of the interface $\sigma_{K, L}$, it was previously defined.

Correction of the diffusion coefficient It is well known that the upwind scheme implicitly introduces too much diffusion. In order to gain more precision when needed, the diffusion coefficient is corrected. This correction is based upon the power law scheme introduced by Patankar[11]. Let $\alpha_{K, L}$ be an approximation of the diffusion coefficient at the interface $\sigma_{K, L}$, it is corrected as follows:

$$
\alpha_{K, L}=\alpha_{K, L} \cdot \max \left(0,\left(1-0.1 \operatorname{Re}_{l}\right)^{5}\right)
$$

where $R e_{l}=\frac{\left|v_{k, L}\right||| X_{K}-X_{L} \|}{\alpha_{K, L}}$ is called the "local Reynolds number". Since $\lim _{h \rightarrow 0} \operatorname{Re}_{l}=0$, the diffusion scheme is still consistent (at the limit, there is no more "correction" of the diffusion coefficient). Furthermore, the stability of the convection scheme is preserved. Here, it is important to understand that this correction does not improve the order of the approximation of the convective term. But, as it will be shown later with the numerical results, the accuracy of the results are effectively improved with this treatment. Furthermore, we want to mention that for compressible flows where shocks can be present, the approximation of the convection scheme would have to be improved with a reconstruction method based on MUSCL type scheme.

\subsection{Boundary conditions}

Let $\sigma$ be a boundary interface. When the boundary condition is a Neuman boundary condition, the numerical diffusion flux $F_{\sigma}^{D}$ is equal to the exact flux. Let $\underline{x}_{\sigma}$ be the intersection of the orthogonal bissectors of the interface $\sigma$. When a Dirichlet boundary $g(\underline{x})$ condition applies, the value $\phi_{\sigma}=$ $g\left(\underline{x}_{\sigma}\right)$ is given at the interface. In this case, the numerical diffusion flux is

$$
F_{\sigma}^{D}=\alpha_{\sigma} \tau_{\sigma}\left(\phi_{\sigma}-\phi_{K}\right)
$$

As for the convective flux, the value of the variable $\sigma$ at the interface is only needed when the fluid is incoming $\left(v_{\sigma}<0\right)$. In this case, the convective flux is given by:

$$
F_{\sigma}^{C}=m(\sigma) v_{\sigma} \phi_{\sigma}
$$


where

$$
\phi_{\sigma}=\left\{\begin{array}{cl}
g\left(\underline{x}_{\sigma}\right) & \text { Dirichlet boundary condition } \\
\frac{F_{\sigma}^{D}}{\alpha_{\sigma} \tau_{\sigma}}+\phi_{K} & \text { Neumann boundary condition }
\end{array}\right.
$$

\subsection{Theoretical results}

Consider the following problem:

$$
\left\{\begin{array}{c}
\nabla \cdot(\underline{v} \phi)-\nabla(\alpha \nabla \phi)=s \\
\text { Boundary conditions }
\end{array}\right.
$$

where $s$ is supposed to be regular enough.

The properties shown in [8] depend on the quality of the triangulation. Meshes of the categories $M_{1}, M_{2}$ and $M_{4}$ (with our treatment) are called "admissible meshes". For these meshes the following properties were shown:

1. Convergence. If the mesh is admissible, the scheme converges to the unique solution of problem (9).

2. Error estimate. If the mesh is an admissible mesh and the unique solution $\phi$ of the problem (9) is regular enough $\left(\phi \in L^{2}(\Omega)\right)$, we have the following error estimate:

$$
\left\|e_{K}\right\|_{L^{2}(\Omega)} \leq C h
$$

where $e_{K}=\phi_{K}-\phi\left(X_{K}\right)$, is the error and $C$ is a positive constant which is independent of the mesh size $h, h$ being the diameter of the largest volume.

\section{Maximum principle.}

Consider the transient problem,

$$
\left\{\begin{array}{c}
\frac{\partial \phi}{\partial t}+\nabla \cdot(\underline{\nu} \phi)-\nabla(\alpha \nabla \phi)=s \\
\text { Boundary conditions }+\quad \text { Initial solution }
\end{array}\right.
$$

the following error estimate was demonstrated in [8] for a first order Euler discretization:

$$
\sqrt{\sum_{K \in \mathcal{T}}\left(\phi\left(X_{K}\right)-\phi_{K}\right)^{2} m(K)} \leq C(h+\delta t),
$$

where $h$ is the diameter of the largest volume, $\delta t$ the time step and $C>0$ a constant independant from the time step and the mesh size $h$.

Remark. For meshes of category $M_{3}$, the approximation of the flux between two control volumes in still consistent. Nevetheless, it is assumed that $\phi$ takes the same value in at least two 


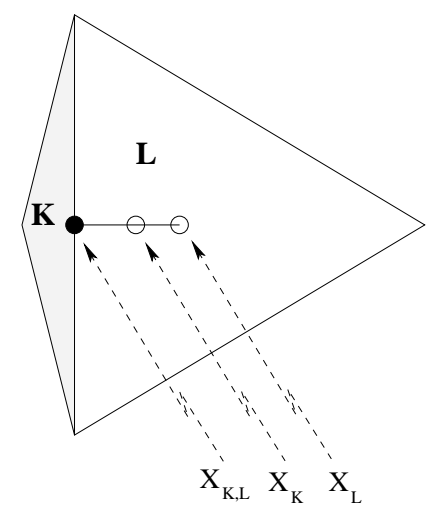

FIG. 3 - Interpolation at an interface

different positions associated to a control volume. In this case, locally, the order of approximation could not be enough to ensure that the latter properties are fulfilled. Neverthless, in [8] it is shown that even for meshes of category $M_{3}$ the scheme can still converge to the true solution when the following condition is fullfiled:

$$
\frac{\sum_{\sigma \in \mathcal{E}} m(\sigma)}{\sum_{\sigma \in \Omega} m(\sigma)} \ll 1,
$$

where $\sigma \in \mathcal{E}$ is the set of interfaces where the flux approximation is not consistent and $\sigma \in \Omega$ is the set of all interfaces.

Remark. In the report[5], the numerical rate of convergence of the diffusion scheme was studied in details. These numerical results show than the observed rate of convergence of the diffusion scheme is of order 2 at the cicumcenters. It is not a contractiction with the theoritical results, it only shows that the theoritical rate of convergence in not optimal.

\subsection{Discrete approximation at an interface}

When physical quantities such as the density or the conductivity are not constant, they may have to be approximated at an interface $\sigma_{K, L}$. Let $\phi$ be a scalar variable and $m(K)$ the measure of control volume $K$ (its volume), the discrete approximation of $\phi$ at the interface $\sigma_{K, L}$ is:

$$
\phi_{K, L}=\frac{m(K) \phi_{K}+m(L) \phi_{L}}{m(K)+m(L)}
$$

The geometrical average allows us to respect locally the minima and the maxima of the solution. Unfortunatly, this approximation gives not as much precision than the linear inteprolation:

$$
\begin{aligned}
\phi_{K, L} & =\left(1-t_{K, L}\right) \phi_{K}+t_{K} \phi_{L} \\
t_{K, L} & =\frac{\left(X_{K}-X_{K, L}\right) \cdot\left(X_{L}-X_{K}\right)}{\left(X_{L}-X_{K}\right) \cdot\left(X_{L}-X_{K}\right)} .
\end{aligned}
$$


This latter interpolation preserves the order of the approximation at the positions $X_{K}$ et $X_{L}$ when $0 \leq$ $t_{K, L} \leq 1$. Nervertheless, in 3D, it is frequent that $t_{K, L} \gg 1$ or $t_{K, L} \ll 0$ (see figure 3 ). With the linear interpolation, the minimum or the maximum of the approximative solution would not be preserved anymore. Preliminary results showed that the linear interpolation could lead to instabilities when the $t_{K, L}$ didn't satisfied the inequality $0 \leq t_{K, L} \leq 1$, which is rather frequent in $3 \mathrm{D}$.

\subsection{Discrete approximation of the gradient}

Since our approximation functions are constant by control volume, the gradient of a function cannot be directly computed (its value being zero over all control volumes). The gradient $(\nabla \phi)_{K}$ of $\phi$ over the volume $K$ is assumed to be constant in all directions. Let $(\nabla \phi)_{\sigma_{k, L}}$ be the normal gradient to the interface $\sigma_{K, L}$ (it being a known quantity), we suppose that the projection of $(\nabla \phi)_{K}$ over $\sigma_{K, L}$ should be closed to $(\nabla \phi)_{\sigma_{k, L}}$ :

$$
\begin{array}{r}
(\nabla \phi)_{K} \cdot \underline{n}_{\sigma_{k, L}} \approx(\nabla \phi)_{\sigma_{k, L}} \\
(\nabla \phi)_{\sigma_{k, L}}=\frac{\tau_{\sigma}}{m(\sigma)}\left(\phi_{L}-\phi_{K}\right)
\end{array}
$$

This equation is then applied to all interfaces which belong to $K$ :

$$
\left[\begin{array}{c}
\underline{n}_{\sigma_{1}} \\
\vdots \\
\underline{n}_{\sigma_{n}}
\end{array}\right]\left[\nabla \phi_{K}\right] \approx\left[\begin{array}{c}
(\nabla \phi)_{\sigma_{1}} \\
\vdots \\
(\nabla \phi)_{\sigma_{n}}
\end{array}\right]
$$

or, in a more compact way:

$$
\underline{\underline{N}}(\nabla \phi)_{K} \approx(\nabla \phi)_{\sigma}
$$

In general, this over-constraint system of equations is not compatible. The "best" solution is approximated with a least square method, the linear system

$$
\underline{\underline{N}}^{t} \underline{\underline{N}}(\nabla \phi)_{K}=\underline{\underline{N}}^{t}(\nabla \phi)_{\sigma}
$$

being solved. It is worth to mention that this approximation is used to calculate the pressure gradient in the momentum equations.

\subsection{Discrete Equations, convection-diffusion operator $\mathcal{C} \mathcal{V}$}

In this section, we discretize the following equation on the domain $\Omega$ :

$$
\frac{\phi^{*}-\phi^{n}}{\delta t}+\nabla \cdot\left(\underline{v}\left(t_{n+1}\right) \otimes \phi^{*}\right)-\nabla\left(\alpha \nabla \phi^{*}\right)=s^{n}
$$

where 
- $\phi$ can be any scalar variable or the components of the velocity vector $\left(v_{x}, v_{y}, v_{z}\right)$;

- $s^{n}$ is a source term;

- $\underline{v}\left(t_{n+1}\right)$ is an approximation of $\underline{v}$ at time $t=t_{n+1}$, it is such that $\nabla \cdot \underline{v}\left(t_{n+1}\right)=0$.

The discrete equations are obtained by integrating (15) over each control volume $K$ and applying the Gauss theorem:

$$
\begin{array}{r}
\int_{K} \frac{\phi^{*}-\phi^{n}}{\delta t} d V+\int_{\partial K}\left(\underline{v}\left(t_{n+1}\right) \otimes \phi^{*}\right) \cdot \underline{n} d S \\
-\int_{\partial K} \nabla\left(\alpha \nabla \phi^{*}\right) \cdot \underline{n} d S=\int_{K} s^{n} d V
\end{array}
$$

The quantities $\phi^{*}, \phi^{n}$ and $s^{n}$ are assumed constant over any given volume $K$. When both the convective and the diffusive schemes are applied to the surface integrals, the discrete equation for $K$ is given by this expression:

$$
\begin{gathered}
m(K) \frac{\phi_{K}^{*}-\phi_{K}^{n}}{\delta t}+\sum_{\sigma \in E_{K}} m(\sigma) v_{\sigma} \phi_{\sigma,+}^{*} \\
-\sum_{\sigma \in E_{K}} \alpha_{\sigma} \tau_{\sigma}\left(\phi_{L}-\phi_{K}\right)=m(K) s_{K}^{n}
\end{gathered}
$$

where $E_{K}$ is the set of interfaces which belong to the boundary of volume $K$. The system (17) is linear but not symmetric. The associated matrix is a diagonal dominant M-matrix.

After, the system (17) is solved for all components of the velocity vector, the solution $\underline{v}^{*}$ is not divergence free. A projection has then to be made.

\subsection{Projection}

Since the flow is incompressible, the continuity equation has to satisfied at all time. In order to compute a velocity field that fulfills this constraint, a projection has to be made. This projection is a combination of two operators: the extension operator $\mathcal{E}$ and the projection operator $\mathcal{P}$.

\subsubsection{Extension operator $\mathcal{E}$}

This operator is applied on each interfaces of the control volumes to compute an intermediate velocity $v_{K, L}^{n+1 / 2}$ :

$$
E:\left(\underline{v}_{K}^{n+1 / 2}, \underline{v}_{K}^{n}, \underline{v}_{L}^{n+1 / 2}, \underline{v}_{L}^{n}\right) \rightarrow\left(v_{K, L}^{n+1 / 2}\right)
$$


To define this operator, we make the assumption that the evolution of this intermediate velocity must agree with the evolution of the velocity computed in the predictor step. Once again, a geometrical average is used:

$$
\begin{array}{r}
v_{K, L}^{n+1 / 2}=v_{K, L}^{n}+\frac{\left[m(K) \delta \underline{v}_{K}+m(L) \delta \underline{v}_{L}\right] \cdot \underline{n}_{K, L}}{m(K)+m(L)} \\
\delta \underline{v}=\underline{v}^{n+1 / 2}-\underline{v}^{n}
\end{array}
$$

When $\sigma$ lies on the domain boundary, the intermediate normal velocity is computed with this expression:

$$
v_{\sigma}^{n+1 / 2}=v_{K}^{n}+m(K) \underline{v}_{K}
$$

It is worth to mention that the boundary condition for the normal velocity must not be considered at this stage. If it were so, it would be possible to construct a non-constant velocity field for which the discrete divergence aprroximation would be zero. In this case, the solution would exibit spurious pressure oscillations that have no physical meaning (false pressure modes).

\subsubsection{Projection operator $P$}

This operator applies to both the pressure and the velocity:

$$
\mathcal{P}:\left(P_{K}^{n}, v_{\sigma}^{n+1 / 2}\right) \mapsto\left(P_{K}^{n+1}, v_{K}^{n+1}\right)
$$

This operation is carried out in two steps. The equation (5) is first written under this form:

$$
\underline{v}^{n+1}=\frac{\delta t}{\beta} \nabla\left(\delta P^{n+1}\right)+\underline{v}^{n+1 / 2} .
$$

This expression is then substitute into the continuity equation which is then discretized:

$$
\frac{\delta t}{\beta} \sum_{\sigma \in E_{K}} m(\sigma)\left(\nabla \delta P^{n+1}\right)_{\sigma}=\sum_{\sigma \in E_{K}} m(\sigma) v_{\sigma}^{n+1 / 2}
$$

The equation(21) enables us to correct the pressure field. After applying equation (20) to all interfaces, the equation

$$
\sum_{\sigma \in E_{K}} m(\sigma) v_{\sigma}^{n+1}=0
$$

is satisfied for all control volumes. Nervertheless, before solving (21), appropriate boundary conditions have to be given. When the normal velocity $v_{\sigma}$ is imposed (at an inlet, a wall or on a symetrie plane), the following Neumann boundary condition holds:

$$
\frac{\delta t}{\beta} \nabla\left(\delta P^{n+1}\right) \cdot \underline{n}=v_{\sigma}^{n+1 / 2}-v_{\sigma} .
$$


The only other case considered is an imposed pressure (such as at an outlet). In this case, the boundary condition for the pressure correction Dirichlet boundary condition:

$$
\delta P_{\sigma}^{n+1}=g\left(\underline{x}_{\sigma}, t^{n+1}\right)-g\left(\underline{x}_{\sigma}, t^{n}\right)
$$

where $g\left(\underline{x}_{\sigma}, t^{n+1}\right)$ is the given pressure at time $t=t^{n+1}$ at the position $\underline{x}_{\sigma}$.

After updating the velocity at all intefaces, a velocity correction is also made on the cells. This last correction has to be compatible with the velocity update that has been made on the interfaces:

$$
\left(\underline{v}_{K}^{n+1}-\underline{v}_{K}^{n+1 / 2}\right) \cdot \underline{n}_{\sigma}=\left(v_{\sigma}^{n+1}-v_{\sigma}^{n+1 / 2}\right)
$$

Let, $W_{\sigma}$ be a parameter such that

$$
W_{\sigma}=\left\{\begin{array}{cc}
\text { big number: (i.e. } \left.10^{6}\right) & \sigma \in \text { wall } \\
1 & \text { otherwise }
\end{array} .\right.
$$

Consider a generic control volume $K$, a linear system of equations is built by applying (23) to all interfaces which belong to $E_{K}$. This linear system can be inconsistent, its solution is always approximated with a least square method. The parameter $W$ has to be introduced in order that the fluid doesn't leave nor enter the domain when a control volume is adjacent to a wall.

Both the velocity-pressure formulation and projection operator fall into the same class than the operators presented in [3]. Hence, the prove of the unicity of the solution for the pressure given in [3] also aplies to this scheme.

\subsection{The complete algorithm}

- Given the initial solution $\underline{v}_{K}^{-1}=0, \underline{v}_{K}^{-1 / 2}, P_{K}^{-1}$ and $\phi_{K}^{0}$, apply the extension and the projection operators:

$$
\begin{array}{r}
\left(P_{K}^{0}, \underline{v}_{K}^{0}\right)=\mathcal{P}\left(P_{K}^{-1}, v_{\sigma}^{-1 / 2}\right) \\
\left(v_{\sigma}^{-1 / 2}\right)=E\left(\underline{v}_{K}^{-1}, \underline{v}_{K}^{1 / 2}, \underline{v}_{L}^{-1}, v_{L}^{1 / 2}\right)
\end{array}
$$

- Given a solution $\underline{v}^{n}, P^{n}$ and $\phi^{n}$,

1. apply the convection-diffusion operator to the velocity field:

$$
\underline{v}_{K}^{n+1 / 2}=\mathcal{C D}\left(\underline{v}_{K}^{n}\right)
$$

2. apply the extension and the projection operators:

$$
\begin{gathered}
\left(v_{\sigma}^{n+1 / 2}\right)=E\left(\underline{v}_{K}^{n+1 / 2}, \underline{v}_{K}^{n}, \underline{v}_{L}^{n+1 / 2}, \underline{v}_{L}^{n}\right) \\
\left(P_{K}^{n+1}, \underline{v}_{K}^{n+1}\right)=\mathcal{P}\left(P_{K}^{n}, \underline{v}_{\sigma}^{n+1 / 2}\right)
\end{gathered}
$$




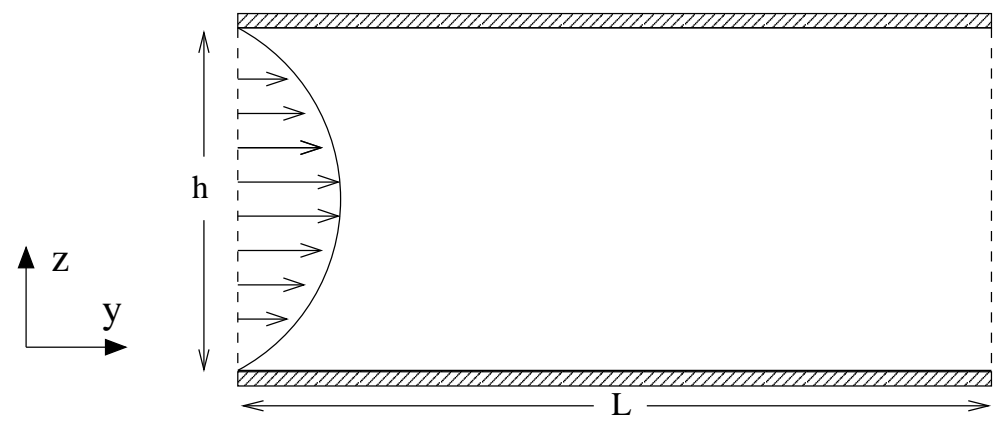

FIG. 4 - Geometry, Poiseuille flow between two parallel planes

3. apply the convection-diffusion operator to all scalar quantities:

$$
\phi_{K}^{n+1}=\mathcal{C D}\left(\phi_{K}^{n}\right)
$$

\section{Numerical results}

In this section, we present some of numerical tests that were conducted to validate this numerical scheme. All computations were carried out in a three dimensional domains, even for the 2D flows.

\subsection{Poiseuille flow}

This is an incompressible flow between two parallel planes with a velocity profil developed at the inlet. The outlet is free and flow in bounded by two walls. The $x$ axes is a symetry plane. The mesh is composed 7200 elements. Which, when combined together give 1200 hexaedras. The initial solutioin and the boundary conditions are the following:

\section{Domain:}

$$
[0.0,0.1] \times[0.0,1.0] \times[0.0,0.2]
$$

\section{Boundary conditions:}

Inlet:

$$
v(y=0, z)=100 z(h-z), \quad w(y=0, z)=0 .
$$

\section{Outlet:}

$$
\left.\frac{\partial v}{\partial n}\right|_{y=L}=0, \quad w(x, y=L, z)=0, \quad P(x, y=L, z)=0
$$


Walls:

$$
\left.\underline{v} \cdot \underline{n}\right|_{z=0, z=h}=0,\left.\quad \underline{v} \cdot \underline{\tau}\right|_{z=0, z=h}=0 .
$$

\section{Initial solution}

$$
\underline{v}(\underline{x})=0, \quad P(\underline{x})=0 .
$$

\section{Physical properties and dimensions:}

$$
R e=\frac{\rho v_{\max } L}{\mu}=100, \quad \rho=1.0, \quad v_{\max }=1, \quad L=1.0, \quad \mu=0.01 .
$$

\section{Time step:}

$$
\delta t=0.1
$$

\section{Convergence critera:}

$$
\left|v^{n+1}-v^{n}\right|_{\infty}<2 \times 10^{-5} .
$$

The analytical solution to this problem is well known and can be stated as follow:

$$
\begin{aligned}
v(y, z) & =100 z(h-z) \\
P(y, z) & =\left.P\right|_{y=L}-\frac{\partial P}{\partial y}(y-L) \\
\frac{\partial P}{\partial y} & =-2
\end{aligned}
$$

Since the exact solution for this problem can be easily computed, we compared our numerical solution with the true solution. The figure 7 shows the maximum norm between the numerical results and the exact solution for the horizontal components of the velocity vector, the horizontal component of the pressure gradient and the pressure. This figure shows that the convergence criteria was satisfied quickly (15 iterations) and that the numerical solution is converging towards the true solution. In order to allow comparaisons, the figure 5 shows the numerical solution near the outlet $(y=0.98)$ and the pressure at the center of the duct $(z=0.1)$ is shown on figure 6 .

\subsection{Lid-driven cavity flow}

This problem deals with a confined flow in a square cavity. The flow is driven by the constant displacement of the upper wall where the tangent velocity is imposed. The settings for this problem are the following: 


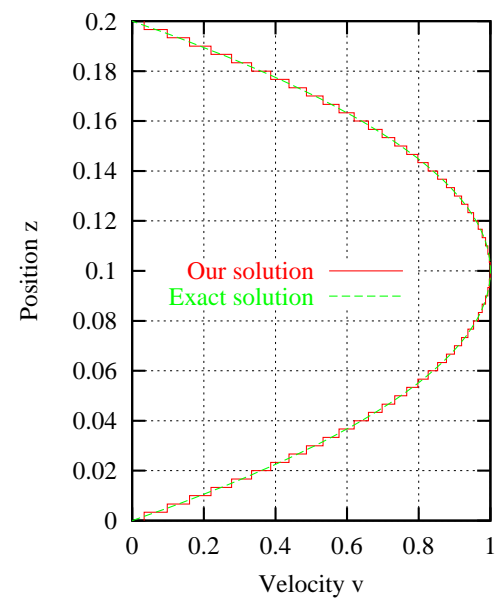

FIG. 5 - Horizontal velocity $v$ near the outlet $(y=0.98)$

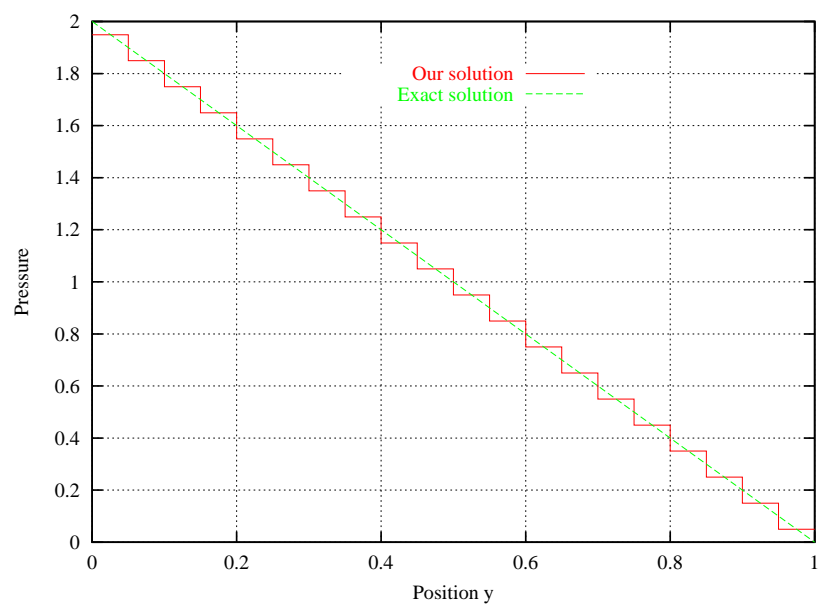

FIG. 6 - Pressure at the mid-plane section $(z=h / 2)$ 


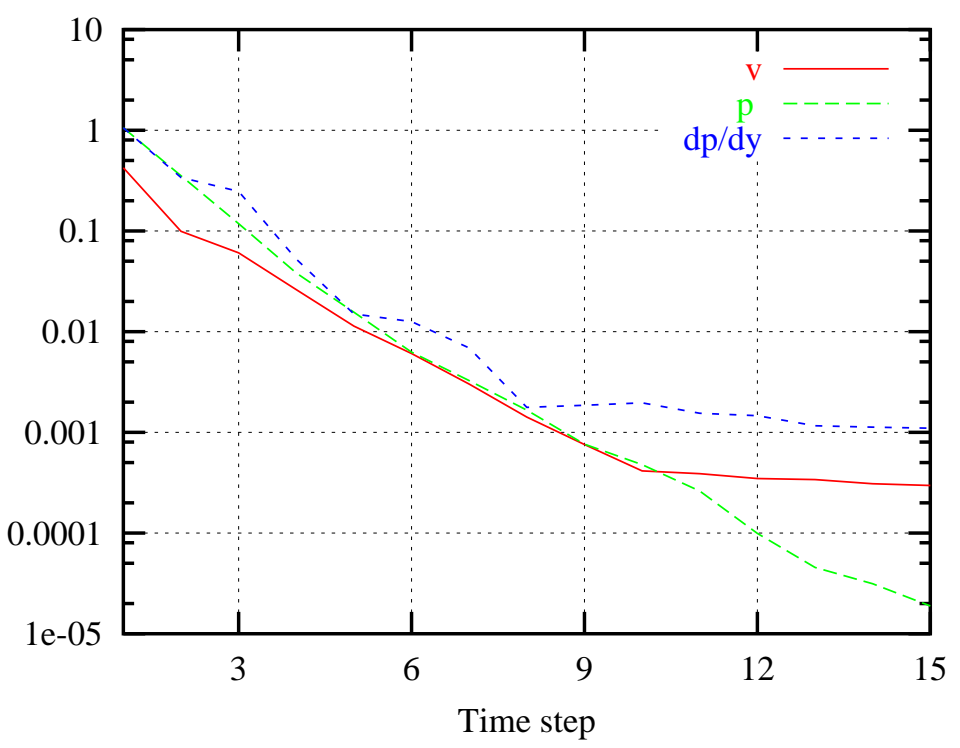

FIG. 7 - Convergence : maximum norms $\left|\phi_{i}^{n+1}-\phi\left(\underline{x}_{i}\right)\right|_{\infty}$ of the error for $v, p$, and $\partial p / \partial y$

\section{Domain:}

$$
[0.0,0.1] \times[0.0,1.0] \times[0.0,1.0]
$$

\section{Boundary conditions:}

$$
\left.\underline{v} \cdot \underline{n}\right|_{\partial \Omega}=0, \quad \underline{v} \cdot \underline{\tau}=\left\{\begin{array}{cc}
1 & z=L \\
0 & \text { elsewhere }
\end{array} .\right.
$$

Initial solution:

$$
\underline{v}(\underline{x})=\underline{0}, \quad P(\underline{x})=0 .
$$

\section{Physical properties:}

$$
R e=\frac{\rho v_{\max } L}{\mu}=1000, \quad \rho=1.0, \quad v_{\max }=1, \quad L=1.0, \quad \mu=0.001 .
$$

\section{Time step:}

$$
\delta t=0.1
$$




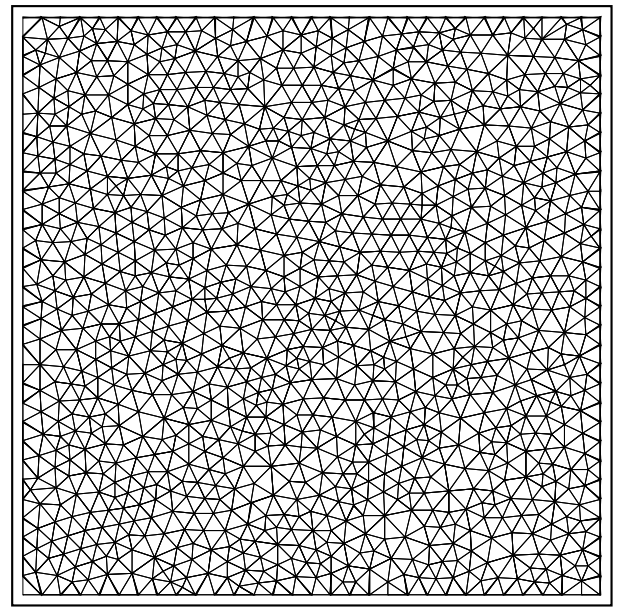

Figure 8: Coarsest mesh for computation of the lid-driven cavity flow (2220 cells)

\section{Convergence critera:}

$$
\left|v^{n+1}-v^{n}\right|_{\infty}<2 \times 10^{-5} \text { and }\left|w^{n+1}-w^{n}\right|_{\infty}<2 \times 10^{-5}
$$

This problem has been studied in details by Ghia and al.[9] for different Reynolds numbers. Hence, our results could be compared to those obtain by others with 2 second order discretization scherme. This problem was solved with three different unstructured meshes made of 2220, 3982 and 6178 control volumes (a plane cut of the coarsest mesh is shown in figure 8). Furthermore, two simulations were carried out for each meshes: one with the power-law scheme, the other without a correction of the diffusion coefficent. The velocity components on the mid-planes were given in [9] and some local characteristics of the flow are presented in table 1. In this table, we show the maximas of the velocity components and locations at which they occur. These results show two things:

1. with mesh refinement, out solution convergs towards the benchmark solution;

2. the quality of the results are improved with the power-law scheme.

In figure 9, we show both the benchmark solution and our numerical solution obtain with with the finest mesh and the power-law scheme.

\subsection{Transient 2D flow around a disc}

This problems deals with an internal flow between two parallel planes. A disc is present near the outlet. The center of the disc is slightly above the mid-section. Hence, the flow is not symmetric 


\begin{tabular}{|c|c|c|c|c|}
\hline Mesh & Power-law scheme & Min. $v(z=)$ & Min. $w(y=)$ & Max. $w(y=)$ \\
\hline \hline 2220 volumes & no & $-0.299,(0.233)$ & $-0.432,(0.883)$ & $0.286,(0.184)$ \\
\hline 2220 volumes & yes & $-0.341,(0.234)$ & $-0.481,(0.883)$ & $0.325,(0.184)$ \\
\hline 3982 volumes & no & $-0.321,(0.233)$ & $-0.448,(0.885)$ & $0.304,(0.184)$ \\
\hline 3982 volumes & yes & $-0.359,(0.202)$ & $-0.492,(0.895)$ & $0.347,(0.158)$ \\
\hline 6178 volumes & no & $-0.351,(0.224)$ & $-0.481,(0.886)$ & $0.337,(0.184)$ \\
\hline 6178 volumes & yes & $-0.387,(0.199)$ & $-0.519,(0.886)$ & $0.373,(0.167)$ \\
\hline \hline Ghia et al.10000 nodes & -- & $-0.383,(0.172)$ & $-0.516,(0.906)$ & $0.371,(0.156)$ \\
\hline
\end{tabular}

ТАВ. 1 - Comparative results for three meshes
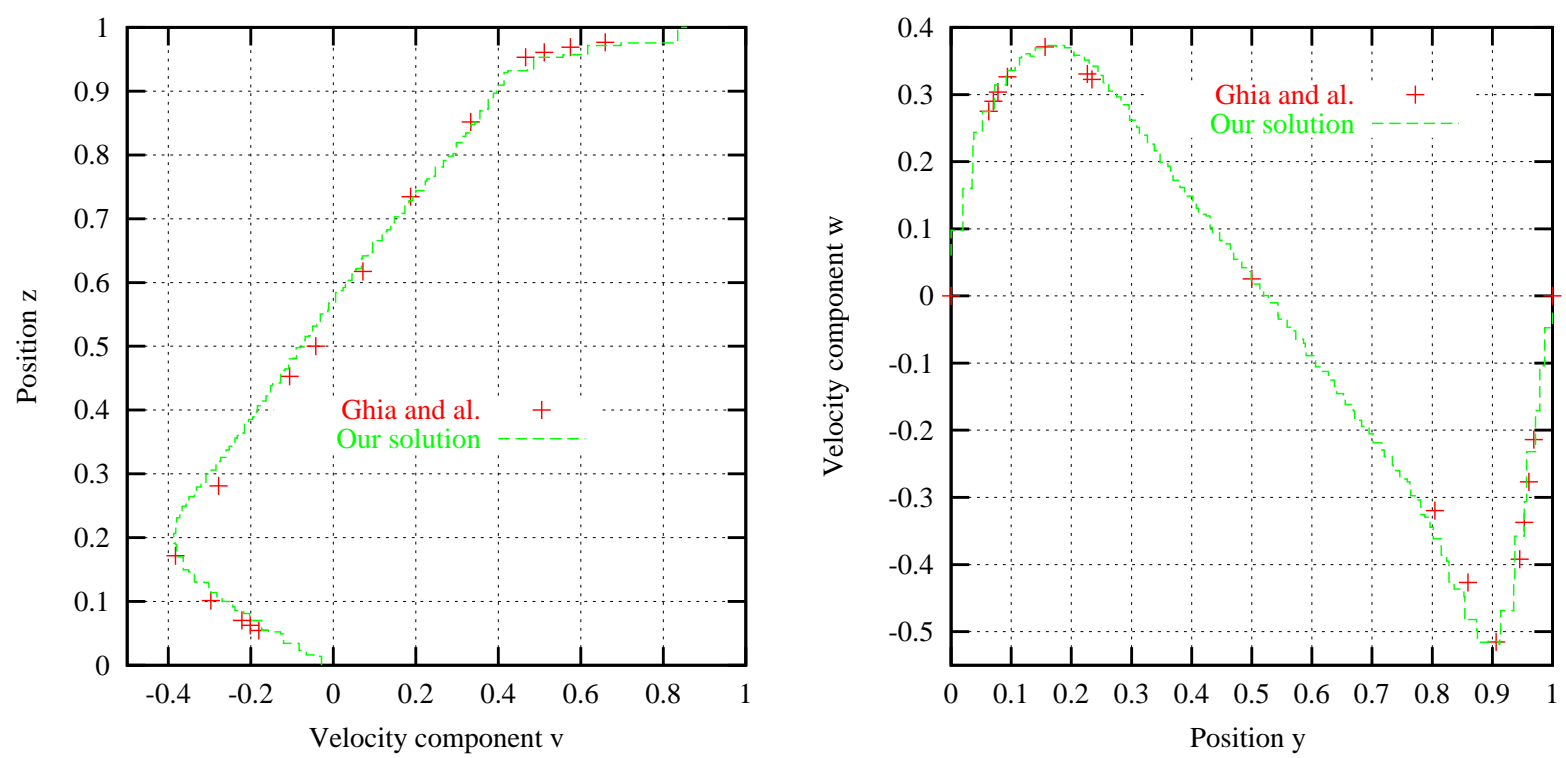

FIG. 9 - Comparative results, velocity components on the mid-planes $y=1 / 2$ and $z=1 / 2$ 

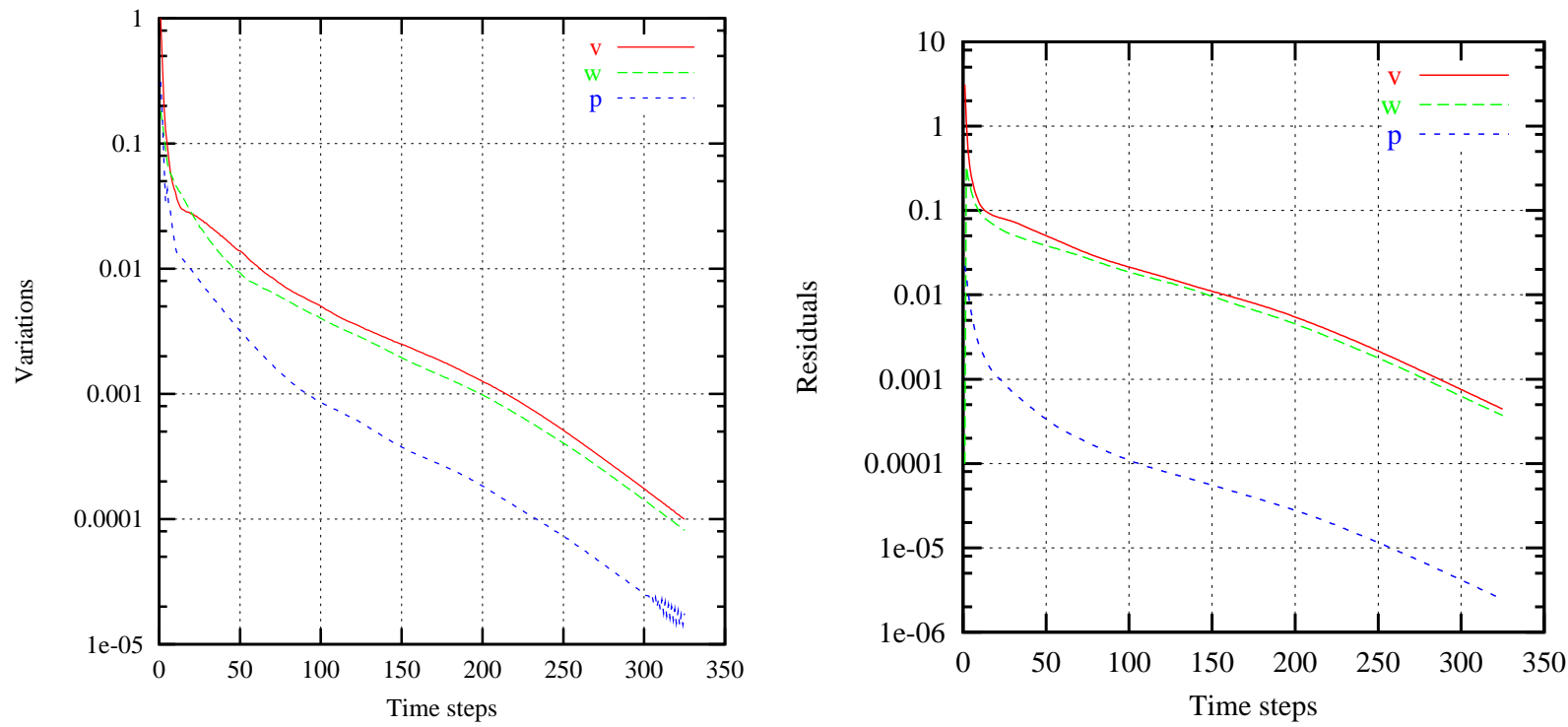

FIG. 10 - Convergence : the graph on the left shows the norms of the variations $\left|\phi^{n+1}-\phi^{n}\right|_{\infty}$, the norms $\left\|\phi^{n+1}-\phi^{n}\right\|_{L^{2}}$ of the residuals are shown on the right

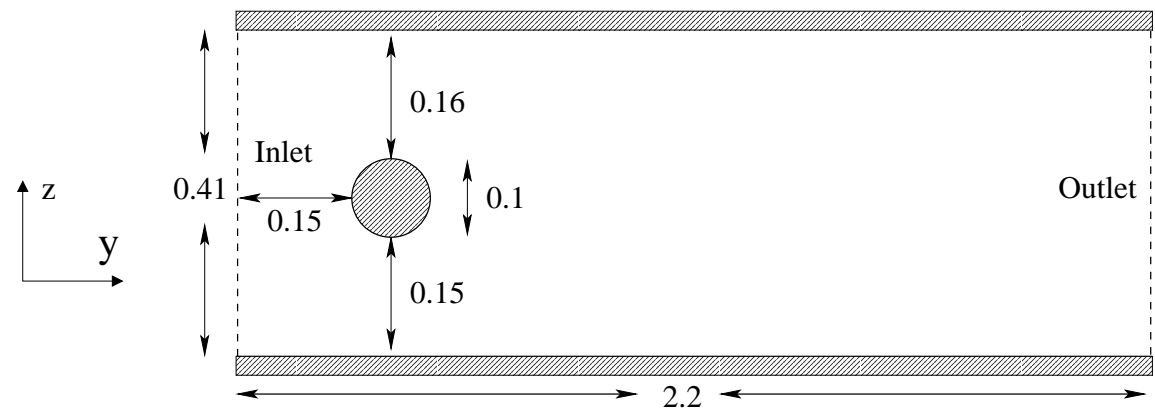

FIG. 11 - Geometry, 2D flow around a disc 
and lift is produced. For this Reynolds number $(R e=100)$, the flow is periodic due to vortex shedding. The settings for this problem are the following:

Domain: See Figure 11.

\section{Boudary conditions:}

Inlet:

$$
v(x, y=0, z)=\frac{4 \cdot v_{m o y} \cdot(H-z)}{H^{2}}, \quad w(x, y=0, z)=0
$$

\section{Outlet:}

$$
\left.\frac{\partial v}{\partial n}\right|_{y=2.2}=0, \quad w(x, y=2.2, z)=0, \quad P(x, y=2.2, z)=0
$$

Walls:

$$
\underline{v} \cdot \underline{n}=0, \quad \underline{v} \cdot \underline{\tau}=0 .
$$

Initial solution:

$$
\underline{v}(\underline{x})=\underline{0}, \quad P(\underline{x})=0 .
$$

Physical properties:

$$
R e=\frac{\rho v_{a} D}{\mu}=100, \quad \rho=1.0, \quad v_{a}=1.0, \quad D=0.1, \quad \mu=0.001
$$

\section{Time steps:}

$$
\delta t=\{0.008,0.004,0.002,0.001\}
$$

For this problem, we compare our results to benchmark quantities published by Turek and al.(see [13]). In this report, the drag, lift, difference of pressure and Strouhal number are presented. Let $t_{0}$ be the time when the maximum value of lift is reached and $f$ the frequency of vortex shedding, the following qantities are provided:

1. drag coeffcient:

$$
\begin{aligned}
C_{D}\left(t_{0}\right) & =\frac{2 \cdot F_{D}\left(t_{0}\right)}{\rho \cdot v_{a}^{2} \cdot D \cdot H \cdot L} \\
F_{D}\left(t_{0}\right) & =\int_{\partial S}\left(0, \mu \frac{\partial v_{\tau}}{\partial n}, P\right) \cdot \underline{\tau} d A ;
\end{aligned}
$$


2. lift coefficient:

$$
\begin{aligned}
C_{L} \quad\left(t_{0}\right) & =\frac{2 \cdot F_{L}\left(t_{0}\right)}{\rho \cdot v_{a}^{2} \cdot D \cdot H \cdot L}, \\
F_{L}\left(t_{0}\right) \quad= & -\int_{\partial S}\left(0, \mu \frac{\partial v_{\tau}}{\partial n}, P\right) \cdot \underline{n} d A ;
\end{aligned}
$$

3. pressure difference:

$$
\Delta P\left(t_{0}+\frac{1}{2 f}\right)=P(0,0.15,0.2)-P(0,0.25,0.2) ;
$$

4. Strouhal number:

$$
S t=\frac{D f}{v_{\text {moy }}}
$$

where:

- $L=0.10$ is the depth in the $3 \mathrm{rd}$ dimension (the flow being resolved in a 3D domain);

- $\underline{\tau}$ is a tangent vector to the cylinder surface;

- $v_{\tau}=\underline{v} \cdot \underline{\tau}$ is the tangential speed at the cylinder surface;

- $\underline{n}$ is the unit normal vector to the surface cylinder;

- $\partial S$ est la surface du disque.

In order to show that we able to obtain a solution independant from the grid size and the time step, computations were carried out on three different meshes and four different time steps. The benchmark quantities provided by Turek and al[13] and our results are presented in table 2 .

\subsection{D flow around a cylinder}

This flow is similar to the previous one. It is a 3D stationary flow around a cylinder confined in a square duct (see figure 12). Once again, we present a complete description of the datas needed to setp-up this problem.

Boudary conditions:

\section{Inlet:}

$$
v(y=0, z)=16 \cdot v_{m} \cdot(H-y)(H-z) / H^{4}, \quad u(y=0, z)=w(y=0, z)=0 .
$$




\begin{tabular}{|c|c|c|c|c|c|}
\hline$\delta t$ & Cells & $\begin{array}{c}C_{D} \\
t=t_{0}\end{array}$ & $\begin{array}{c}C_{L} \\
t=t_{0}\end{array}$ & $S t=\frac{D \cdot f}{v_{m}}$ & $\begin{array}{c}\Delta P \\
t=t_{0}+\frac{1}{2 f}\end{array}$ \\
\hline \hline 0.008 & 22188 & 3.254 & 0.973 & 0.278 & 2.448 \\
\hline 0.004 & 22188 & 3.217 & 0.942 & 0.281 & 2.426 \\
\hline 0.002 & 22188 & 3.203 & 0.924 & 0.284 & 2.416 \\
\hline 0.001 & 22188 & 3.200 & 0.919 & 0.283 & 2.419 \\
\hline 0.008 & 32110 & 3.268 & 1.018 & 0.284 & 2.494 \\
\hline 0.004 & 32110 & 3.226 & 0.987 & 0.287 & 2.476 \\
\hline 0.002 & 32110 & 3.212 & 0.962 & 0.287 & 2.474 \\
\hline 0.001 & 32110 & 3.206 & 0.952 & 0.287 & 2.476 \\
\hline 0.008 & 46259 & 3.278 & 1.028 & 0.297 & 2.502 \\
\hline 0.004 & 46259 & 3.243 & 1.025 & 0.294 & 2.487 \\
\hline 0.002 & 46259 & 3.230 & 1.009 & 0.292 & 2.485 \\
\hline 0.001 & 46259 & 3.228 & 1.006 & 0.292 & 2.495 \\
\hline \hline & & $3.22-3.24$ & $0.99-1.01$ & $0.295-0.305$ & $2.46-2.50$ \\
\hline
\end{tabular}

TAB. 2 - Maximum drag, lift and other comparative results

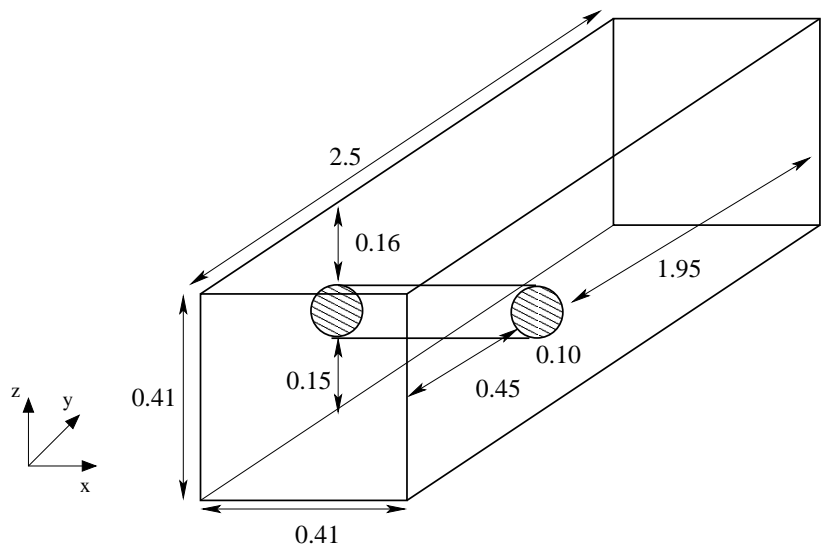

Figure 12: Geometry, 3D flow past a cylinder 


\section{Outlet:}

$$
\left.\frac{\partial v}{\partial n}\right|_{y=2.5}=0, \quad w(y=2.5, z)=0, \quad P(y=2.5, z)=0
$$

\section{Walls:}

$$
\underline{v} \cdot \underline{n}=0, \quad \underline{v} \cdot \underline{\tau}=0 .
$$

Initial solution:

$$
\underline{v}(\underline{x})=\underline{0}, \quad P(\underline{x})=0 .
$$

Physical properties and dimensions:

$$
\operatorname{Re}=\frac{\rho v_{m} D}{\mu}=20, \quad \rho=1.0, \quad v_{m}=0.45, \quad D=0.10, \quad \mu=0.001, \quad H=0.41 .
$$

\section{Time step:}

$$
\delta t=0.1
$$

\section{Convergence critera:}

$$
\left|u^{n+1}-u^{n}\right|_{\infty}<1 \times 10^{-4},\left|v^{n+1}-v^{n}\right|_{\infty}<1 \times 10^{-4} \text { and }\left|w^{n+1}-w^{n}\right|_{\infty}<1 \times 10^{-4} .
$$

As for the previous flow, this problem is part of the bechmark problems presented in [13]. As for the transient flow, the solutions were not provided. Instead, the lift coefficient, the drag coefficients and the pressure variation between two points were. These quantities can be computed with these expressions:

$$
\begin{array}{r}
F_{D}=\int_{\partial S}\left(\mu \frac{\partial v_{\tau}}{\partial n} n_{z}-P n_{y}\right) d S \\
F_{L}=-\int_{\partial S}\left(\mu \frac{\partial v_{\tau}}{\partial n} n_{y}+P n_{z}\right) d S \\
C_{D}=\frac{2 F_{D}}{\rho v_{m o y}^{2} D H}, \quad C_{L}=\frac{2 F_{L}}{\rho v_{m o y}^{2} D H} \\
\Delta P=P(0.45,0.20,0.205)-P(0.55,0.20,0.205)
\end{array}
$$

$S$ is the surface of the cylinder, $\underline{n}$ the normal unit vector on $S$ and $v_{\tau}$ the tangent speed at the surface of the cylinder. 


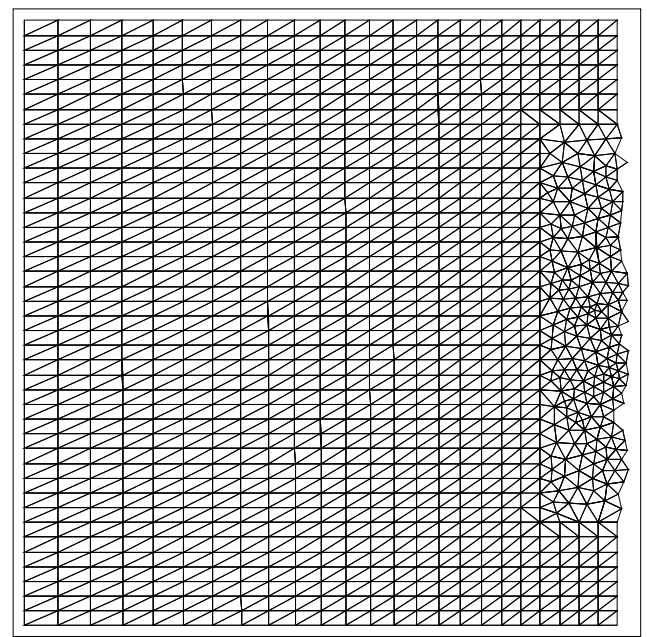

Figure 13: Mesh at the inlet

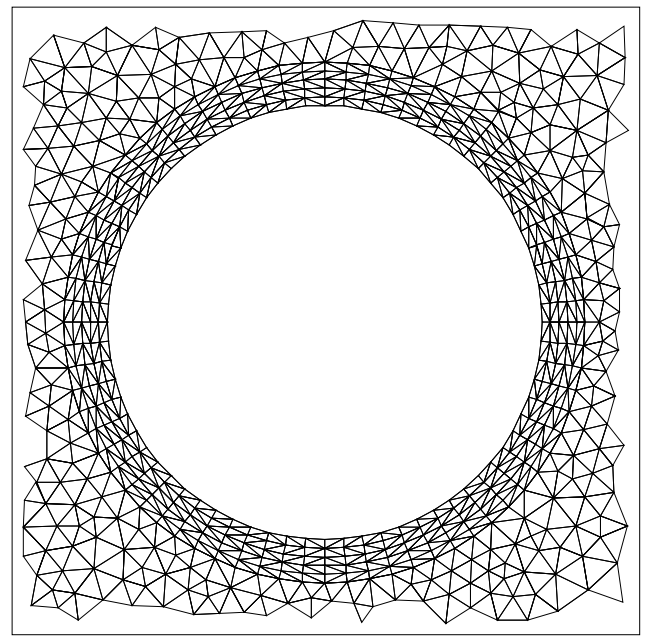

Figure 14: Mesh around the cylinder 


\begin{tabular}{|c|c|c|c|c|}
\hline & Unknowns & $C_{L}$ & $C_{D}$ & $\Delta P$ \\
\hline \hline Mesh 1 & 646000 & 0.0115 & 6.201 & 0.170 \\
\hline Mesh 2 & 794000 & 0.0105 & 6.186 & 0.170 \\
\hline Mesh 3 & 1069000 & 0.00824 & 6.180 & 0.170 \\
\hline \hline Benchmark & 753664 to & 0.008 to & 6.05 to & 0.165 to \\
quantities & 12582912 & 0.010 & 6.25 & 0.175 \\
\hline
\end{tabular}

TAB. 3 - Lift, drag for 3D flow around a cylinder
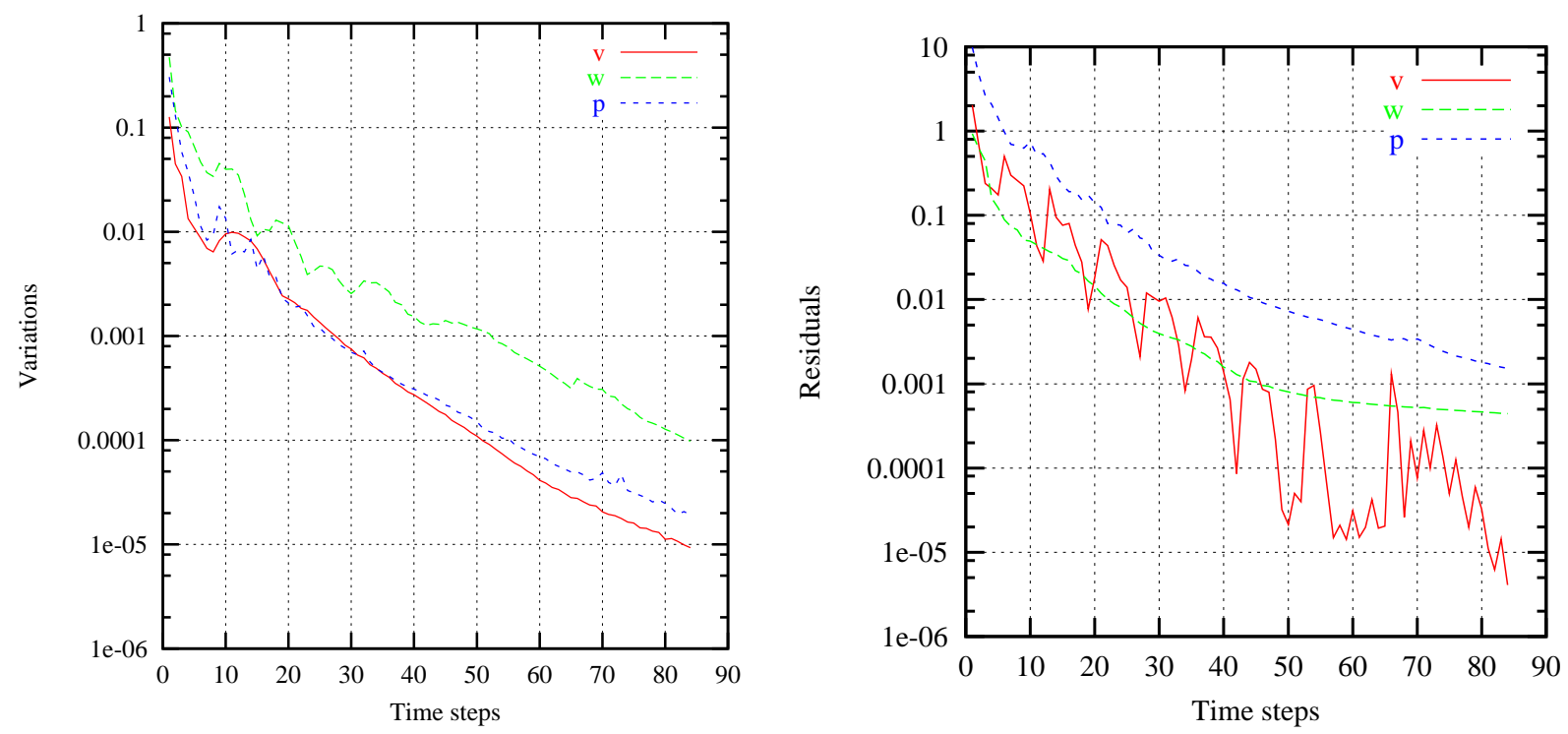

FIG. 15 - Convergence : the graph on the left shows the norms of the variations $\left|\phi^{n+1}-\phi^{n}\right|_{\infty}$, the norms $\left\|\phi^{n+1}-\phi^{n}\right\|_{L^{2}}$ of the residuals are shown on the right

In order to make a thoughfull validation, three computations were carried out on different meshes. The 3D meshes were built by translating a 2D mesh composed of cut-quadrangles and triangles. We may recall that, locally, semi-structed meshes fall into $M_{3}$ and $M_{4}$ category of meshes. Hence, in this regions, the mesh used for the computations is composed of hexaedras (the tetrahedras being combined together). At the inlet, around the cylinder and at the outlet, the grid is a semi-structured mesh and the control volumes are hexahedras. Further from the cylinder, the mesh is completly unstructured. Parts of the 2D mesh used to built the second 3D mesh are shown on figures 13 and 14 .

In table 3, the references quantities obtain with our scheme are compared to those presented by Turek[13] . Our results are in very good agreement with the benchmark solutions. As for the other permanent flow problems, we show both the convergence curves for the residuals and the variations of all variables. 


\subsection{D thermal flow in a cylinder}

This probem deals with a forced thermal flow in a cylinder where the temperature is imposed both at the inlet and on the cylinder's surface. The flow is not developped at the inlet, a constant velocity being imposed at this location. All the datas needed to solve this problem are presented below.

\section{Domain:}

$$
\partial \Omega=\left(\begin{array}{c}
x(\theta) \\
y(\theta) \\
z
\end{array}\right)=\left(\begin{array}{c}
0.05 \cdot \cos (\theta) \\
0.05 \cdot \sin (\theta) \\
z
\end{array}\right), \quad 0 \leq \theta \leq \pi / 2, \quad 0 \leq z \leq 1.5
$$

\section{Boundary conditions:}

\section{Inlet:}

$$
\begin{array}{cl}
u(x, y, z=0)=0, & v(x, y, z=0)=0, \quad w(x, y, z=0)=1 \\
& T(x, y, z=0)=1
\end{array}
$$

\section{Outlet:}

$$
\begin{array}{ll}
u(x, y, z=1.5)=0, \quad v(x, y, z=1.5)=0, & \left.\frac{\partial w}{\partial n}\right|_{z=1.5}=0 \\
P(x, y, z=1.5)=0, & \left.\frac{\partial T}{\partial n}\right|_{z=1.5}=0 .
\end{array}
$$

\section{Walls:}

$$
\underline{v} \cdot \underline{n}=0, \quad \underline{v} \cdot \underline{\tau}=0, \quad T=0 .
$$

\section{Initial solution:}

$$
\underline{v}(\underline{x})=\underline{0}, \quad P(\underline{x})=0, \quad T(\underline{x)}=0 .
$$

Physical properties and dimensions:

$$
\begin{array}{r}
R e_{D}=\frac{\rho w_{m} D}{\mu}=100, \quad w_{a}=1.0, \quad \rho=1.0, \\
\operatorname{Pr}=\frac{\mu c_{p}}{k}=2.0, \quad D=0.1, \quad c_{p}=1.0 .
\end{array}
$$




\section{Time step:}

$$
\delta t=0.1
$$

\section{Convergence critera:}

$$
\left|w^{n+1}-w^{n}\right|_{\infty}<2 \times 10^{-5} .
$$

$w_{a}$ is the average of the speed for a section of the duct and $D$ the diameter of the duct. This flow being symetric, the computations were only carried out on one quarter of the domain. The 3D mesh was built using the extrusion of a 2D mesh composed of triangles. The 3D mesh was made of 45920 cells divided in 40 sections.

For this problem, there is an analytical solution in the region of the domain where the flow is fully developed. For a laminar flow, $\left(\operatorname{Re}_{D} \preceq 2300\right)$, the length at which the flow is fully developed is given by this expression:

$$
\left(\frac{L}{D}\right)_{\text {lam }} \approx 0.05 R e_{D} .
$$

In this region, the velocity component $w$ and the pressure gradient can be computed from these equations:

$$
\begin{aligned}
w\left(r=\sqrt{x^{2}+y^{2}}\right) & =-\frac{1}{4 \mu} \frac{\partial P}{\partial z}\left(\frac{D}{2}\right)^{2}\left(1-\left(\frac{r}{D / 2}\right)^{2}\right), \\
\frac{\partial P}{\partial z} & =-\frac{8 \cdot \mu \cdot w_{m}}{(D / 2)^{2}} .
\end{aligned}
$$

As for the temperature, the length at which the flow is thermally developed is given by this expression:

$$
\left(\frac{L}{D}\right)_{l a m, T} \approx 0.05 R e_{D} \cdot \operatorname{Pr} .
$$

In this region, we don't have an algebraic expression for the temperature. Nevertheless, in this region, the is no variation along the cylinder of the dimensionless temperature:

$$
\frac{\partial}{\partial z}\left(\frac{T_{s}-T(\underline{x})}{T_{s}-T_{m}(z)}\right)=0,
$$

$T_{m}(z)$ being the mean axial temperature in a given section.

In figures 16 to 17 we show some of the results. In figure ??, we show that there is no significant difference between the computed velocity component $w$ and the analytical solution given by equation 24 . The figure 17 compares the pressure gradient component $\partial P / \partial z$ to the exact solution given by equation 25 . It is obvious that, in the fully developed flow region, the pressure gradient behaves according to the exact solution: it is constant both in the axial and longitudinal directions 


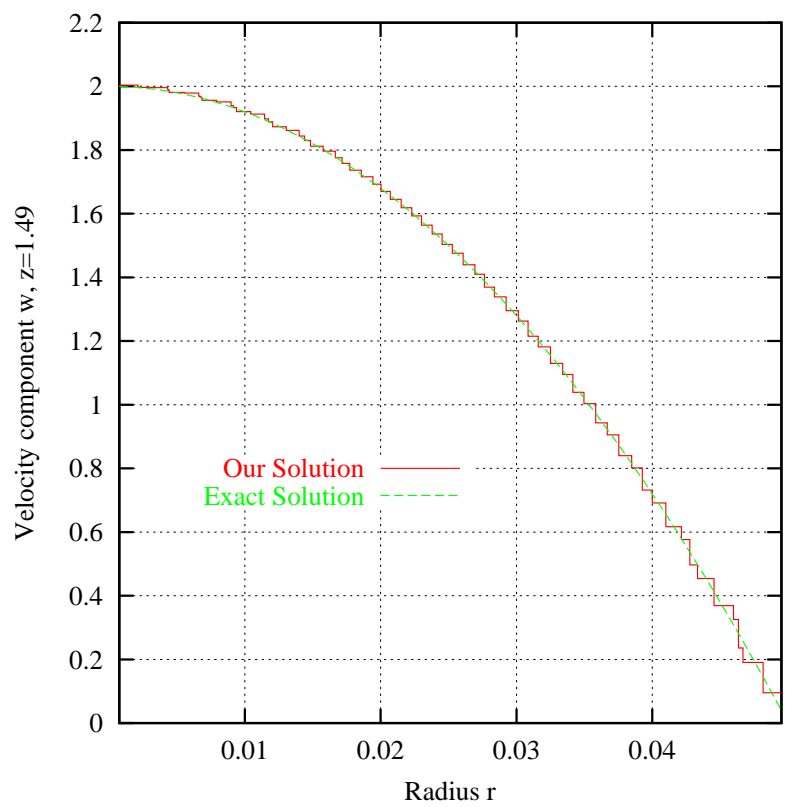

FIG. 16 - Velocity profile near the outlet

and its value is very close to the exact value-3.2. The figure 18 gives a general idea of the adimensional temperature behavior. Near the center of the duct $(r=0.4)$, the adimensional temperature still show slight variations. As for the other stationary flows, we show the convergence curves in figure 19.

\section{Conclusion}

A solver for the Navier-Stokes equations has been proposed to solve incompressible viscous flows and the convection-diffusion of scalar quantities. This solver is based upon a fractional step scheme and the finite volume method on unstructured meshes. This solver is one a the few which allow the local conservation of mass and scalar quantities and the numerical preservation of the maximum principal for scalar quantities.

The convection-diffusion scheme is very robust and easy to implement. Nevertheless, being of order one, it is a low order scheme. The convection scheme accuracy's could be improved with a more sophisticated method such as MUSCL.

Numerical solutions for laminar stationary and non-stationary flows were presented. For all cases, the solutions computed with this scheme were in good agreement with those presented by other researchers or exact solutions. For all stationary flows, we showed the convergence curves for both the variations and the residuals. This scheme is under active development, numerical 


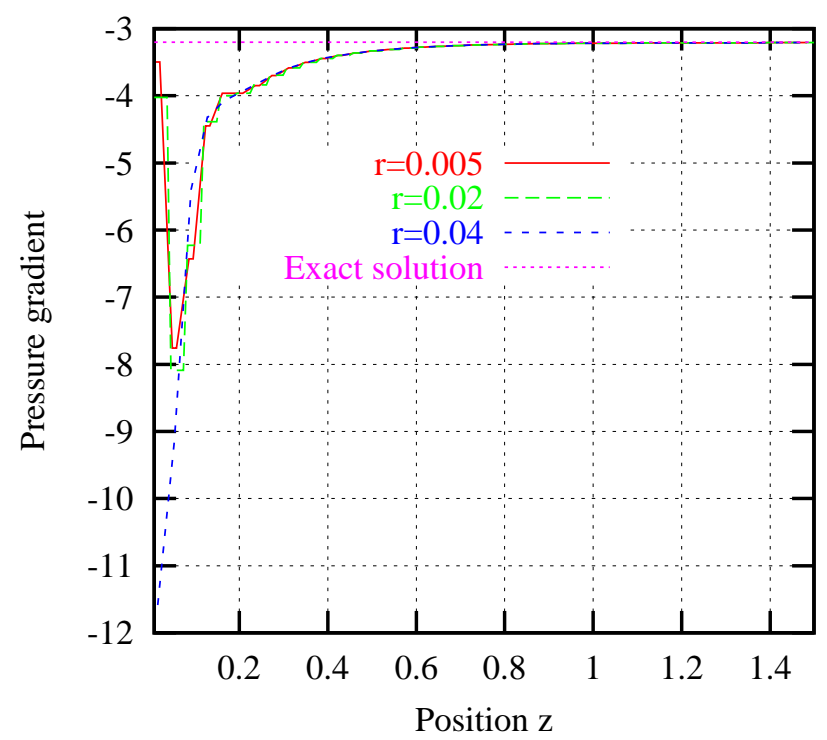

FIG. 17 - Pressure gradient along the duct, component $\partial P / \partial z$

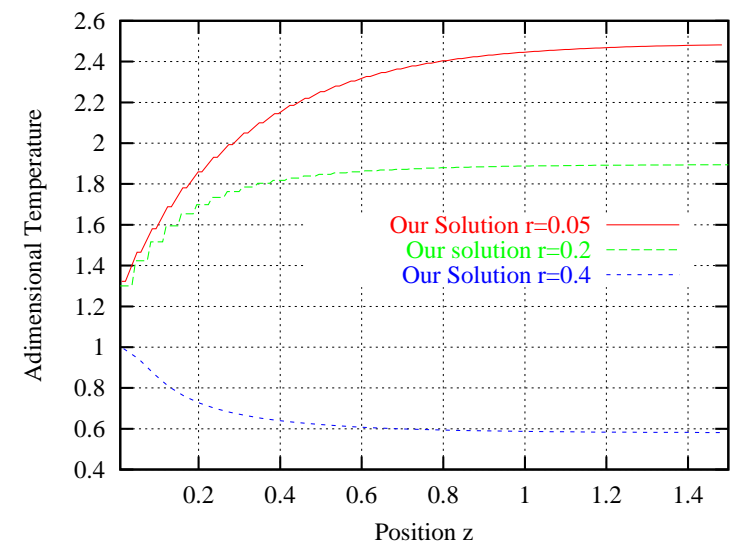

FIG. 18 - Adimensional temperature profile along the cylinder 

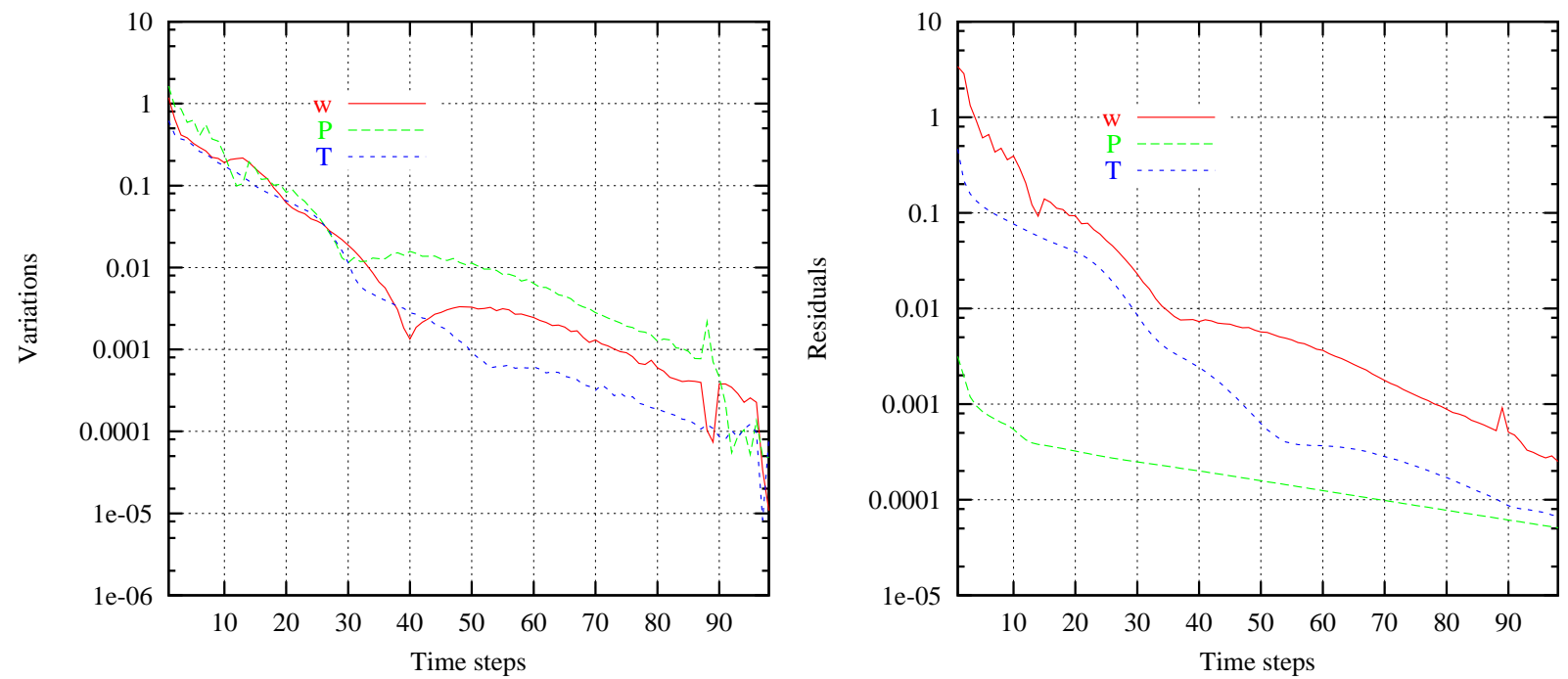

FIG. 19 - Convergence : the graph on the left shows the norms of the variations $\left|\phi^{n+1}-\phi^{n}\right|_{\infty}$, the norms $\left\|\phi^{n+1}-\phi^{n}\right\|_{L^{2}}$ of the residuals are shown on the right

results for a turbulent flow using the $k-\varepsilon$ model have already been presented in [4, 12]. Further developments of the scheme include: a second order approximation of the convection scheme, more exhaustive computations of turbulent flows with heat transfer and extension to compressible flows.

\section{References}

[1] S. Boivin, F. Cayré, and J.M. Hérard. A finite volume method to solve the Navier-Stokes equations for incompressible flows on unstructured meshes. International Journal of Thermal Science, 39:806-825, 2000.

[2] S. Boivin, F. Cayré, and J.M. Hérard. A finite volume scheme to compute incompressible gas-solid two-phase flows. American Institute of Aeronautics and Astronautics, AIAA-2000$2665,2000$.

[3] S. Boivin and J.M. Hérard. Un schéma de volumes finis pour résoudre les équations de Navier-Stokes sur une triangulation. Revue Européenne des Éléments Finis, 5:461-490, 1996.

[4] S. Boivin, J.M. Hérard, and S. Perron. A finite volume method to solve the navier-stokes equations for incompressible flows on unstructured meshes. In Proceedings, 9th Annual Conference of the CFD Society of Canada, page 56, 2001. 
[5] F. Cayré. Schémas volumes finis pour un problème elliptique sur un maillage triangulaire, étude numérique de convergence. Technical Report HE-41/97/057/A, Électricité de France, Direction des Études et Recherche, 1997.

[6] F. Cayré. Méthodes de volumes finis pour maillages non structutés pour la simulation numérique des Écoulements incompressibles monophasiques et diphasiques. Master's thesis, Université Laval, 1999.

[7] A.J. Chorin. Numerical solution of the Navier-Stokes equations. Mathematics of Computation, 22:745-762, 1968.

[8] R. Eymard, T. Gallouet, and R. Herbin. Finite Volume Methods. Hanbook of Numerical Analysis, 7, 2001.

[9] U. Ghia, K. N. Ghia, and C. T. Shin. High-Re solutions for incompressible flow using the Navier-Stokes equations and a multigrid method. Journal of Computational Physics, 48:387411, 1982.

[10] J.M. Lopez and J. Shen. Numerical simulation of incompressible flows in cylindrical geometries using a spectral projection method. International Journal of Applied Science And Computing, 5:25-40, 1998.

[11] S. V. Patankar. Numerical Heat transfer and Fluid Flow. McGraw-Hill, 1980.

[12] S. Perron. Résolution numérique d'écoulements 3 dimension avec une nouvelle méthode de volumes finis pour maillages non structurés. $\mathrm{PhD}$ thesis, Université du Qu'ebec à Chicoutimi, 2001.

[13] M. Schafer and S. Turek. Benchmark computations of laminar flow around cylinder. In Proceedings DFG Priority Research Program, Flow Simulation on High Performance Computers. Vieweg, 1992.

[14] J. Shen. On error estimates on projection methods for Navier-Stokes equations: first order schemes. SIAM Journal Numerical, 29:55-77, 1992.

[15] J. Shen. On error estimates on projection methods for the Navier-Stokes equations: second order schemes. Mathematics of computation, 65:1039-1065, 1996. 